\title{
PROMOCIÓN DE LA SALUD Y EDUCACIÓN FÍSICA ESCOLAR. DISCURSOS, ACCIONES Y PRETENSIONES EN INSTITUCIONES EDUCATIVAS DE MEDELLÍN*
}

\author{
PROMOTION OF SCHOOL HEALTH AND PHYSICAL EDUCATION. \\ SPEECHES, ACTIONS, PRETENDED IN EDUCATION INSTITUTIONS IN MEDELLÍN
}

\author{
Víctor Alonso Molina Bedoya** \\ Juan José Quintero Castrillón*** \\ Sebastián Restrepo Ramírez****
}

\section{Resumen}

\begin{abstract}
El artículo presenta los resultados alusivos a la articulación de los direccionamientos de las instituciones educativas de Medellín y los encaminamientos de los organismos responsables de la política pública en el campo de la educación física, el deporte y la recreación desde una intención por la promoción de la salud. La investigación ha tenido como propósito diseñar de manera participativa en las instituciones educativas de Medellín una propuesta de indicadores de gestión que articulen necesidades e intereses de carácter local y global relacionados con la promoción de la salud. La investigación se inscribe en el enfoque de la investigación acción participativa en educación y se aborda a partir de dos momentos: deconstrucción y reconstrucción, las herramientas han sido talleres colectivos y encuestas. Se ha evidenciado una apuesta por la promoción de la salud en las instituciones, pero se demanda igualmente de indicadores cualitativos y/o cuantitativos, al igual que de acciones reflexivas para la construcción de verdaderos proyectos de escuelas saludables.
\end{abstract}

Palabras clave: promoción de la salud, indicadores, educación, higiene (fuente: DeCS, BIREME).

\section{Abstract}

This paper presents the outcomes referring to the management articulation in education in Medellin, and the perspective of the authority responsible for public policies in physical education, sport and recreation fields, as an intention for health promotion at schools. The purpose of this research is to design a management indicators proposal which assembles global and local, needs and interests related to health promotion in a joint effort with educative Institutions in Medellin. With a Participatory Action Research (PAR) approach, this research is placed within two tensions: deconstruction and reconstruction. Workshops and surveys tools have been used, in which evidence has shown the importance of health promotion in institutions. It equally requires qualitative and quantitative indicators, from reflexive constructive actions to the implementation of real healthy schools projects.

Keywords: health promotion, indicators, education, public health (source: DeCS, BIREME).

Fecha de recepción: 12 de septiembre de 2013

Fecha de aprobación: 28 de marzo de 2014

* Artículo asociado a la investigación "Construcción participativa de indicadores de gestión para promover una cultura de hábitos y estilos de vida saludable fundamentados en el desarrollo humano, la convivencia y la paz en las Instituciones Educativas de Medellín y el área metropolitana", financiada por la Universidad de Antioquia y la Funlam, Medellín, Colombia, 2013.

** Doctor por la Universidad de Valladolid, España. Grupo de investigación "Ocio, expresiones motrices y sociedad". Instituto de Educación Física. Universidad de Antioquia. Correo electrónico: vmolina@catios.udea.edu.co

*** Licenciado en Educación Física. Correo electrónico: juanjoudea1989@hotmail.com

**** Licenciado en Educación Física. Correo electrónico: restre1031@hotmail.com 


\section{INTRODUCCIÓN}

El artículo permite una lectura de los principales asuntos levantados a partir de la investigación "Indicadores de gestión para promover una cultura de hábitos y estilos de vida saludables en el sector escolar" y de las intenciones constatadas en la segunda fase en desarrollo: "Construcción participativa de indicadores de gestión para promover una cultura de hábitos y estilos de vida saludable fundamentados en el desarrollo humano, la convivencia y la paz", ejercicio que opta metodológicamente por el enfoque de la investigación acción participativa (IAP), la cual se inscribe dentro del marco de la investigación cualitativa, misma que procura romper con la relación simple entre sujeto y objeto y así poder establecer un diálogo con la comunidad que permita generar aprendizaje mutuo y transformación de las realidades.

De acuerdo con el objeto de problematización, y luego de la revisión teórica, el equipo de investigación ha abordado las categorías promoción de la salud y estilos de vida saludables. La primera, entendida como práctica y teoría social comprometida con la dignificación de la existencia de los individuos y los colectivos humanos. Desde Breilh (2003), se comprende que los problemas determinantes de las enfermedades no solo se generan en los organismos individuales, y tampoco se encuentran exclusivamente en el cuerpo humano, por el contrario, son procesos que se originan en los diferentes modos en que la sociedad se organiza, y de manera especial, en los particulares patrones de trabajo y de consumo.

Por su parte, los estilos de vida saludables se asumieron como un proceso dinámico y dialéctico configurado en la dimensión social por la cual adquiere presencia la reproducción. Por ella, se expone una comprensión y vivencia individual relacionada con determinados patrones/modelos de vida.

\section{OBJETIVO}

Diseñar de manera participativa en las instituciones educativas (IE) del área metropolitana del municipio de Medellín una propuesta de indicadores de gestión para el área de la educación física, la recreación y el deporte en el sector escolar, articulando necesidades e intereses de carácter local y global relacionados con la promoción de la salud, desde la perspectiva del desarrollo humano, la convivencia y la paz.

\section{MATERIALES Y MÉTODOS}

A nivel metodológico, se optó por un enfoque de investigación acción participativa, la cual se inscribe dentro del marco de la investigación cualitativa y procura romper con la relación simple entre sujeto y objeto con el propósito de producir colectivamente conocimiento a partir de una relación horizontal. Con este tipo de investigación, se pretende que la realidad se transforme, teniendo en cuenta a los actores sociales. Según Rojas (2002), "la investigación y la ciencia deben estar al servicio de la colectividad; busca ayudarle a resolver sus problemas y necesidades y ayudar a planificar su vida" (p. 2).

La investigación se inscribe en el enfoque de la investigación acción participativa en educación. Para el caso de este proyecto de investigación, se pretende elaborar un diseño curricular general por competencias con la participación de la comunidad educativa de las IE seleccionadas del área metropolitana del municipio de Medellín. Se retomó el tipo de "investigación acción educativa (IAE), de corte pedagógico"1, el cual, según Restrepo (2004), apoyándose en los trabajos de Stenhouse (1993) y Elliot (1994), considera que la IAE está más ligada a la transformación de procesos escolares en general, mientras que la investigación acción pedagógica (IAP) está más focalizada en la práctica pedagógica de los docentes.

En tal sentido, y atendiendo a los principios básicos de la investigación acción (Murcia, 1991), se integraron los momentos de deconstrucción y reconstrucción una vez articuladas las fases de reflexión sobre un área problemática, la planeación y la ejecución de acciones alternativas para mejorar la situación problemática y la evaluación de resultados con miras a emprender nuevos ciclos a partir de las tres fases, a la manera de evaluación permanente.

El trabajo se ha realizado a partir de talleres colectivos con los actores educativos de las distintas instituciones y de la aplicación y análisis de encuestas. La información ha sido recolectada de 21 centros educativos. Para la fase de análisis, se ha realizado lectura cruzada de la información obtenida en los talleres con las entrevistas y los documentos institucionales.

\footnotetext{
1 El momento de la evaluación será objeto de un nuevo proyecto de investigación.
} 


\section{RESULTADOS Y DISCUSIÓN}

\section{Derechos sociales, discurso oficial y saberes hegemónicos de la salud escolar}

Para esta discusión sobre las articulaciones entre las políticas sectoriales y los enrutamientos educativos de las IE, se asumieron los derechos sociales como los aquellos que hacen posible al ciudadano una participación mínima en la riqueza material y espiritual lograda por la colectividad (Coutino, citado por Flausino y Mascarenhas, 2012). Se conviene entonces en que el deporte, el ocio y la educación física son derechos de los ciudadanos que deben ser garantizados a partir de las políticas sociales públicas como un bien común, público, democrático y de acceso universal para el conjunto de la población.

Se habla de tal suerte, y de acuerdo con la indagación realizada, de una primera crítica a la garantía de tales derechos sociales para el grueso de la sociedad colombiana, y esto tiene que ver con las acciones y los direccionamientos del Estado hacia políticas de focalización, más que de universalización en la prestación y concretización de los derechos como responsabilidad estatal.

No em tanto, em um país que se desobriga da garantia das condições mínimas de sobrevivência, os referidos direitos não são efetivados por diversos motivos. Dentre eles estão, a ocorrência de políticas focais que priorizam localidades com vulnerabilidade social contrapondo-se à proposta de universalização, parcos financiamentos que não atendem as demandas, inexistência de um controle democrático e o recuo do Estado com o avanço das políticas neoliberais (Flausino y Mascarenhas, 2012, p. 3).

Como se puede observar, la estrategia de la focalización en la garantía de los derechos ciudadanos resulta ser una alternativa de los gobiernos para atender estados de máxima vulnerabilidad social generados por fuertes procesos de ruptura en el tejido social, sin atender las causas estructurales que producen tales situaciones.

Esta focalización bien se puede leer en las IE desde tres puntos de vista muy confluyentes entre sí. El primero se refiere a la alta presencia del discurso de los derechos al deporte, la educación física y la recreación por parte de las comunidades educativas sin distingo alguno; y que a la vez se constituyen en ejes direccionadores de la actuación institucional. El segundo punto hace referencia a la baja financiación de que disponen las IE para la ejecución de programas, planes y proyectos que permitan a los estudiantes un acceso real a las oportunidades que brinda la práctica a su desarrollo integral como persona y como sujeto social. Y, por último, está la comprensión de nuestro sector como un medio efectivo para la consecución de otras finalidades sociales como la paz, la convivencia y la superación del conflicto en una suerte de mistificación o instrumentalización de lo que somos y de lo que hacemos.

\section{LOS DISCURSOS}

En los discursos institucionales relacionados con la actividad física de los últimos años, prevalece una reinterpretación del área, inspirada en la premisa de actuación y ajuste a los nuevos tiempos de cambio de siglo. Así, se asiste a la emergencia de un reconocimiento de las prácticas relacionadas con la educación física, la recreación y el deporte en las IE, donde se apropian, reconceptualizan y recontextualizan los discursos y las prácticas. En esta dirección:

No es suficiente la adquisición de destrezas o el perfeccionamiento de nuevas formas de movimiento, sino que se requiere integrar a la formación del estudiante diversos conocimientos que lo hagan competente para actuar de manera apropiada y flexible en contextos variados y cambiantes" (Ministerio de Educación Nacional, 2010, p. 12).

Una recurrencia en las orientaciones pedagógicas del MEN tiene que ver con los fundamentos epistemológicos que soportan el área: el cuerpo, el movimiento y el juego. Al respecto, se puede decir que, si bien aparentemente estos conceptos se han transformado por otros como los de corporeidad, motricidad y lúdica, se infiere que la nueva connotación que han adquirido estos conceptos es muy cercana a la apropiación que se hace en Colombia de la teoría pedagógica de la educación física de Ommo Grupe (1976), el cual se apoya en autores como Merleau Ponty y que hace visible una concepción formativa donde lo corporal alude a la unidad dialéctica cuerpo-movimiento como condición de posibilidad de ser, estar y actuar en el mundo. Y donde el juego es condición de posibilidad de lo lúdico como ejercicio de la libertad y de la creatividad humana.

Igualmente, se definen, además de los asuntos objeto de intervención y de formación, nuevas metas para el campo. Preocupaciones sociales por la salud, la violen- 
cia, el conflicto social, la calidad de vida, la sostenibilidad del ambiente, la defensa de los derechos, la reivindicación de la diversidad tocan nuestra área de desempeño.

Desde las orientaciones pedagógicas del MEN se resalta:

Promover el cultivo personal desde la realidad corporal del ser humano, aportar a la formación del ser humano en el horizonte de su complejidad, contribuir a la construcción de la cultura física y a la valoración de las expresiones autóctonas, apoyar la formación de la cultura ciudadana y de valores primordiales para la convivencia y la paz, apoyar una educación ecológica, desarrollar una pedagogía de la inclusión para todas las personas en las prácticas de la educación física, la recreación y el deporte.

Intenciones que armonizan con las pretensiones del Plan Decenal del Deporte, la Recreación, la Educación Física y la Actividad Física 2009-2019:

En 2019, Colombia será una nación reconocida por el impacto que generan las políticas y programas en deporte, recreación, educación física y actividad física sobre el desarrollo social y económico del país. El apoyo a estas actividades se reflejará en mejores indicadores de convivencia y de salud pública en la población en general y en mayores logros en el deporte de alto rendimiento, que consoliden al país como potencia deportiva a nivel continental (2009, p. 9).

Con este plan se busca, por un lado, atender la obligación constitucional y brindar un horizonte indicativo para el deporte, la recreación, la educación física y la actividad física, a la vez que evaluar las experiencias y las políticas que se han impulsado desde el Estado.

No obstante estas intenciones y cambios del campo acorde con los nuevos tiempos, evidenciadas en los documentos institucionales del MEN y del Plan Decenal del Deporte, la Actividad Física, la Recreación y la Educación Física, y la normal reapropiación e interpretación de las IE, en lo concerniente a la intención por la salud en la escuela, se constata un discurso muy ligado a la higiene y la terapeutización de nuestras prácticas en el ámbito escolar, como se demostrará a continuación.

\section{VISIÓN HIGIÉNICA Y TERAPÉUTICA DE LA SALUD ESCOLAR ${ }^{2}$}

Una orientación que se develó en los discursos de las IE y que emergió como una categoría de análisis para el equipo de investigación fue la visión higiénica y terapéutica de la educación física. En algunos enunciados se lee:

Higiene y salud (IEB, 2011); " primeros auxilios (signos vitales; lesiones en huesos, articulares, musculares; intoxicaciones, heridas, hemorragias, quemaduras". (IEB, 2011); "beneficios de una buena postura corporal, corrección postural, lesiones articulares y tratamiento (IEB, 2011).

La educación física se concibe así como un medio para la salud (Prinz et al., 2007), pero además como un dispositivo para la formación en valores, para los derechos, los deberes, la educación corporal, la lúdica y para la apropiación de prácticas culturales de la actividad física.

Objetivos del área: aportar a los actores del proceso educativo, en el contexto de sus intereses, necesidades de salud, derechos, deberes y responsabilidades individuales y sociales, a través del conocimiento, la valoración, expresión y desarrollo de la dimensión corporal, la dimensión lúdica y la enseñanza de la diversidad de prácticas culturales de la actividad física (IEM, 2012) ${ }^{4}$.

Esta orientación adquiere relevancia en un interés social y educativo que permita evidenciar y constatar un manejo responsable del propio cuerpo a partir de la formación de hábitos y costumbres sanas de higiene corporal en las IE de la región, aspecto enunciado en los direccionamientos del MEN, órgano estatal encargado de la garantía y la vigilancia del sistema educativo colombiano y que traduce las aspiraciones planteadas en la Constitución Política de 1991 (2002). Sobre este interés estatal por un manejo adecuado del cuerpo en las IE del país, es clara la relación entre la política y la categoría social cuerpo, irrebatible en las observaciones de Galantini (2001), en sus producciones hegemónicas en torno al cuerpo, en que se fijan las bases territoriales para el mercado capitalista, donde se requiere de un poder que organice la circulación de cosas y de perso-

\footnotetext{
2 Ver más ampliamente sobre este tema lo desarrollado por Molina (2013).

3 Institución educativa del municipio de Bello.

4 Institución educativa del municipio de Medellín.
} 
nas. Nicos Poulantzas (1980) considera que el cuerpo no es solo una naturalidad biológica, sino, y sobre todo, una institución política. Para Galantini, (2001), hay una clara connivencia entre lo corporal y lo político.

Al respecto, en un apartado se lee: "adquirir hábitos y costumbres sanas de higiene corporal, en relación con la práctica de actividades físicas, recreativas y deportivas, demostrando una actitud responsable con el manejo del propio cuerpo" (IEM, 2012).

Es oportuno señalar que, aun cuando en algunas IE hay orientaciones por un enfoque crítico y contextualizado de la salud concebida como bienestar y calidad de vida, a la hora de operativizar los documentos y proponer los contenidos, las metodologías, los planes de trabajo y las unidades de clase se observa un desarrollo ligado inextricablemente a la concepción u orientación higiénica (Faleiros y Faleiros, 2008), biomédica y biomecánica del campo y, por tanto, tradicional de la salud. Veamos algunos separados referidos a esto: "flexión, extensión, hiperextensión, abducción, dorsiflexión, oposición, reposición, circunducción, rotación, rotación interna, calentamiento, resistencia, fuerza, coordinación, equilibrio, agilidad, frecuencia cardiaca, presión arterial, etc. (IEM,2012).

Como se observa, hay expresa una tensión entre la salud funcional y la higiene corporal con el de vida humana propiciada o potenciada desde la pedagogía de las expresiones motrices para la promoción y disfrute de las capacidades humanas. De acuerdo con Molina (2004), es necesario un nuevo pensar de la educación física incorporando renovados y diferentes objetos de reflexión epistémica, para trascender los fines y objetivos de aprendizaje centrados básicamente en el desarrollo de habilidades y capacidades motrices y proponer, en cambio, concepciones más amplias y complejas del mundo, la formación y del ser humano. Esta opción teórica, conceptual y metodológica establece una crítica fuerte a la concepción mecánica, instrumental y repetitiva del saber de la educación física.

Una posible explicación de la tensión antes indicada quizás tenga que ver con el aún poco desarrollo de otras nociones al interior del campo de saber, lo que hace que los actores interesados en mirar el objeto de estudio de una manera diferente terminen cayendo en la misma e histórica comprensión del sector (Arboleda, 2011). Para el caso, como se pudo apreciar en los aná- lisis, impera una concepción físico-funcional o valoración físico-biológica, que define como contenidos básicos de la formación lo físico y lo perceptivo como medios propicios para la conservación de la salud y la apropiada conciencia mente-cuerpo (IEM, 2012).

Aunque se evidencia una preocupación por la salud en las IE de las ciudades estudiadas, no se identificaron orientaciones, enrutamientos claros por una comprensión amplia y compleja de esta categoría que la ubique en una dimensión por el carácter multidimensional del salud ligada a la persona como globalidad, su contexto y los determinantes socio-históricos del bienestar humano, social y ambiental (Granda, 1991). La salud tiene que ver con decisiones, actitudes, organizaciones, políticas, espacios, ambiente del centro educativo, actividades, limpieza, calidad espacial y locativa, zonas verdes, relaciones horizontales y participación activa de todos los miembros (Restrepo, 2001), en la edificación de un proyecto educativo integrador e integrado no solo en su interior, sino también con la ciudad, el país y las dinámicas mundiales que determinan el modo de estar y de ser en la actualidad (Emiliozzi, 2011). Se está de acuerdo entonces en que es necesaria una conciencia crítica para adoptar y mantener estilos de vida realmente activos donde las reflexiones sobre los procesos y enfoques de aprendizaje del estudiantado tienen un rol determinante (González, Del Rincón y Del Rincón, 2011, p. 287).

\section{MISTIFICACIÓN}

Como ya se indicó anteriormente, hay una explicita valoración del sector como un medio efectivo para la obtención de otras finalidades de carácter social más generales como la paz, la convivencia, la recuperación económica y la superación del conflicto; algo que se puede interpretar, como lo han hecho ya otros investigadores, como mistificación o instrumentalización del campo por parte de un determinado sector de la sociedad nacional.

Para desarrollar este planteamiento, algunas afirmaciones textuales del Plan Decenal son útiles. En el separado referido al resumen se lee:

La participación del deporte, la recreación, la educación física y la actividad física en la economía, la movilización ciudadana que genera en torno a los eventos, el entusiasmo colectivo qué éstos gene- 
ran, el valor que adquiere el triunfo para la emulación de la disciplina, la promoción de los valores esenciales para la convivencia, las alegrías y tristezas que los deportistas nos entregan, son factores de cohesión social que ayudan a definir nuestro carácter y nuestra vitalidad como nación. Su promoción, práctica y disfrute, es decir, su presencia activa en la población, constituyen indicadores que contribuyen a establecer el grado de felicidad y de plenitud de las personas (2009, p. 11).

Estos cuatro componentes del campo de la cultura corporal son reconocidos en el Plan Decenal como estrategias para el desarrollo del país por cuanto:

1. Contribuyen a la generación de empleo y al desarrollo económico.

2. Son estrategias eficaces en la búsqueda de la paz, la convivencia, la reconciliación y el bienestar social.

3. Promueven el desarrollo cultural, social y ambiental del país.

4. Garantizan los derechos sociales y la inclusión social (2009, p. 16).

En general, se afirma desde el Plan que es una estrategia nacional para el desarrollo humano, la convivencia y la paz. Reconociendo las particularidades de la educación física, la recreación y el deporte en sus formas de expresión e intervención, pero siempre orientadas hacia el bien común; compromiso de la política pública nacional.

El Plan destaca la necesidad de pensar a la nación articulada a las dinámicas de la globalización, donde se considera que, si bien las políticas sociales nacionales gozan de un margen de autonomía, los desafíos de apostarle a la superación del conflicto, la exclusión y la consecución del desarrollo5 son también aspiraciones de la comunidad internacional.

Ahora bien, como se puede apreciar, tanto en los lineamientos del Plan como en las orientaciones pedagógicas del MEN, se reconocen una funcionalidad atribuida al sector, como reparador de lo social; tarea que es coincidente con la asignada por la investigadora Rubiela Arboleda (2004, p. 95) al deporte en Colombia

\footnotetext{
5 Varios estudios en Colombia dan cuenta de la necesidad de una valoración social del sector que busque un compromiso con el desarrollo personal y comunitario (Ver en Carreño y Robayo, 2010).
}

en su análisis de las expresiones motrices, como mecanismo para restablecer la dinámica social.

No obstante, la misma autora devela que ni las expresiones motrices en general, ni el deporte en especial representan un asunto de primer orden en las estrategias políticas de los planes estatales en el continente suramericano. Afirma más precisamente la investigadora:

No existen propuestas educativas que convoquen a la población y brinden alternativas estructurales de cara a las expresiones motrices y con ello al deporte como factor determinante del desarrollo integral de los sujetos y de la calidad de vida de la sociedad (Arboleda 2004, p. 93).

Es inocultable, entonces, el acentuado papel asignado al campo por parte de los discursos oficiales, los órganos de gobierno y los medios masivos de comunicación; mientras que el horizonte de posibilidad real para el ejercicio y aprovechamiento de ellos, como derecho para el conjunto de la sociedad, es hilarante. Ese alto valor, asignado como medio para la paz, la convivencia, el desarrollo humano, la promoción de la salud, el crecimiento económico, la formación ciudadana, el entusiasmo colectivo, la promoción de valores, la disciplina, la formación y la cohesión social son negados al no hacer parte prioritaria de las agendas de gobierno, como política social pública. Esta retórica de los derechos, de los altos y poderosos beneficios de su práctica, sumada a la baja inversión, permiten hablar de fuertes procesos de mistificación e instrumentalización del sector.

Se habla aquí de mistificación en el sentido atribuido por Piacentini (citado en Flausino y Mascarenhas, 2012, p. 9): por cuanto se configura socialmente una ilusión, un engaño, una falsedad, una fantasía, en este caso, alrededor del tema de los derechos a la práctica de la actividad física, el deporte y la recreación.

De acuerdo con Piacentini (citado en Flausino y Mascarenhas, 2012):

(...) Mistificar tem a ver com ilusão, falsidade, fantasia, sendo a mistificação em si o próprio engano. Tal assertiva é verificada no discurso oficial, pois o mesmo é contraditório ao afirmar que o esporte e o lazer são direitos sociais a serem usufruídos por todos universalmente ao mesmo tempo em que se afirma a "utilização" do esporte e do lazer para remediar o uso de drogas, a criminalidade, a violência nas populações em situação de vulnerabilidade social (p. 9). 
Se puede afirmar, según lo anterior, que el alto valor social atribuido al sector como estrategia para contribuir al desarrollo humano, la salud, la convivencia y la paz, por parte de los organismos estatales; la baja inversión real en programas y proyectos, y el poco acompañamiento a las IE dan cuenta de, por un lado, una instrumentalización del campo y, por otro, de su mistificación como derecho social y como parte de una política social pública.

\section{CONCLUSIONES}

Se encontró una correspondencia entre los discursos de las IE, las orientaciones pedagógicas del MEN y las intenciones del Plan Decenal del Deporte.

Una categoría que emergió del trabajo fue la de mistificación, con la cual se quiere sugerir un modo de leer lo que acontece en las instituciones sobre la aplicación de los derechos sociales, en este caso particular, con el derecho al deporte, al ocio, el tiempo libre y la educación física; si bien las orientaciones gubernamentales son precisas, las instituciones son las que, a su buen juicio y posibilidad, tratan de satisfacer este derecho a los estudiantes. En la gran mayoría de las instituciones estudiadas, no se dispone de recursos para el ofrecimiento proyectos y programas en pro del mejoramiento de la calidad de vida de las comunidades educativas.

Se valora para finalizar que, además de la relevancia de los elementos estructuradores de los indicadores de gestión para promover una cultura de hábitos y estilos de vida saludables, esta línea de investigación debe ser fortalecida a partir de investigaciones que impacten la cotidianidad de la escuela y que potencien las capacidades humanas de los educandos. Es preciso reconocer que la escuela aún reproduce una organización que privilegia determinados saberes en procura de un modelo de hombre y de sociedad que no siempre va en la búsqueda de la realización de los sujetos. Los patrones de eficacia y eficiencia se imponen, las más de las veces, sobre la integralidad de la educación y sobre la construcción de sujetos libres, lo cual demanda de la escuela como organización social y política, de una lectura crítica de la forma como se organiza la vida, la cultura, lo social y lo ambiental en un momento particular de la historia (Chinchilla, 2012, p. 6).

\section{AGRADECIMIENTOS}

A la Universidad de Antioquia, a la Funlam, a la USB por la financiación del proyecto. A las instituciones educativas por haber permitido el acceso a sus documentos institucionales.

\section{REFERENCIAS BIBLIOGRÁFICAS}

Arboleda, R. (2011). La tercera ruta: una apuesta metodológica de indagación en torno al cuerpo y la motricidad. Revista Educación Física y Deporte, 30, (1), 355-364.

Arboleda, R. (2004). Las expresiones motrices en América Latina en la tensión global-local: una apuesta desde la corporeidad, en el marco de la modernidad reflexiva. Revista Apunts: Educación Física y deportes, (78), 91-97.

Breilh,J.(2003). Epidemología crítica. Ciencia emancipadora e interculturalidad. Buenos Aires: Lugar Editorial S.A.

Carreño, J.M. y Robayo, N. (2010). Formación en recreación en Colombia: aspectos fundamentales para el estudio. Revista Lúdica Pedagógica, 2, (15), 17- 26.

Chinchilla, V. (2012). Pedagogía, prácticas corporales y subjetivación: pasado y presente de la educación física, el deporte y la recreación escolar. Revista Lúdica Pedagógica, 7 (17), 5-7.

Constitución Política de Colombia. (2002). Bogotá: Intermedio.

Elliot, J. (1994). La investigación-acción en educación. Madrid: Ediciones Morata.

Emiliozzi, M. (2011). Fragmentos de cuerpos despojados: una arqueología y una genealogía de los discursos del cuerpo en el diseño curricular de la educación física. Revista de Educación Física y Deportes, 30, (2), 675-678.

Faleiros, V., y Faleiros, E. (2008). Uma visão histórica da violência contra crianças e adolescentes. En V. Faleiros, \& E. Faleiros, Escola que protege: enfrentando a violência contra criancas e adolescentes (pp. 15-26). Brasilia: Ministerio da Educacao.

Flaustino, M.y Mascarenhas, F. (2012). 0 direito ao esporte e lazer: apontamentos críticos à sua mistificação. Revista Licere, 15, (2), 1-26.

Galantini, G. (Mayo de 2001). Cuerpo y salud en la modernidad: origen del surgimiento de la educación física. Recuperado de www.efdeportes.com/indic36.htm

Granda, E. (1991). El compromiso social de la investigación social en salud. En O. Betancourt, J. Breilh y E. Granda, Reproducción social y salud (pp. 40-58). Guadalajara: Editorial Universidad de Guadalajara. 
González, G. (2011). Estructura latente y consistencia interna del R-SPQ-2F: Reinterpretando los enfoques de aprendizaje en el EEES. Revista de Investigación Educativa, 29, (2), 277-293.

Grupe, O. (1976). Estudios sobre una teoría pedagógica de la educación física. Madrid: Instituto Nacional de Educación Física.

Ministerio de Cultura. (2009). Plan Decenal del Deporte, la Recreación, La Educación Física y la Actividad Física. Bogotá: Coldeportes.

Ministerio de Educación Nacional. (2010). Orientaciones pedagógicas para la Educación Física, Recreación y Deporte. Bogotá.

Molina, V. (2004). Promoción de la salud desde la pedagogía de la motricidad. Recuperado de www. efedeportes.com/efd78/motric.htm

Murcia, J. (1991). Investigar para cambiar, un enfoque sobre investigación acción participante. Bogotá: Cooperativa Editorial del Magisterio.
Poulantzas, N. (1980). Estado, Poder y Socialismo. Madrid: XXI siglo veintiuno editores.

Prinz, A., Drexsler, G. y Werle, V. (2007). Didática da educación física e inclusión. Revista Brasileira de Ciências do Esporte, 28, 103-119.

Restrepo, B. (2004). La investigación-acción educativa y la construcción de saber pedagógico. Revista Educación y Educadores, 7, 45-55.

Restrepo, H. (2001). Agenda para la acción en promoción de la Salud. En H. Restrepo y H. Málaga, Promoción de la salud: Cómo construir vida saludable (pp. 34-55). Bogotá: Editorial Médica Internacional.

Rojas, J. (26 de Mayo de 2009). Investigación acción participativa (IAP). Recuperado de http://quintoisrael. blogspot.com/2009/05/jose-raul-rojas-sdb.html

Stenhouse, L. (1993). La investigación como base de la enseñanza. Madrid: Ediciones Morata. 